\title{
Making oneself predictable: reduced temporal variability facilitates joint action coordination
}

\author{
Cordula Vesper $\cdot$ Robrecht P. R. D. van der Wel • \\ Günther Knoblich $\cdot$ Natalie Sebanz
}

Received: 31 January 2011 / Accepted: 16 April 2011 / Published online: 10 May 2011

(C) The Author(s) 2011. This article is published with open access at Springerlink.com

\begin{abstract}
Performing joint actions often requires precise temporal coordination of individual actions. The present study investigated how people coordinate their actions at discrete points in time when continuous or rhythmic information about others' actions is not available. In particular, we tested the hypothesis that making oneself predictable is used as a coordination strategy. Pairs of participants were instructed to coordinate key presses in a two-choice reaction time task, either responding in synchrony (Experiments 1 and 2) or in close temporal succession (Experiment 3). Across all experiments, we found that coactors reduced the variability of their actions in the joint context compared with the same task performed individually. Correlation analyses indicated that the less variable the actions were, the better was interpersonal coordination. The relation between reduced variability and improved coordination performance was not observed when pairs of participants performed independent tasks next to each other without intending to coordinate. These findings support the claim that reducing variability is used as a coordination strategy to achieve predictability. Identifying coordination strategies contributes to the understanding of the mechanisms involved in real-time coordination.
\end{abstract}

Keywords Temporal coordination · Joint action . Coordination strategy $\cdot$ Predictability

C. Vesper $(\varangle) \cdot$ R. P. R. D. van der Wel · G. Knoblich · N. Sebanz Donders Institute for Brain, Cognition and Behavior, Center for Cognition, Radboud University,

P.O. Box 9104, 6500 HE Nijmegen, The Netherlands e-mail: c.vesper@donders.ru.nl

\section{Introduction}

People perform actions together to reach shared goals (Clark 1996; Sebanz et al. 2006). Successfully performing joint actions often requires that individual actions are precisely coordinated in space and time. In some cases, coordinating with others calls for continuous adaptation between coactors, such as when two people carry a heavy table together and coordinate the forces they apply at each side of it. In other cases, particular actions need to be coordinated at specific points in time such as when two people juggle together and must catch each others' balls.

Prior research has identified two key mechanisms subserving temporal coordination. First, the tendency for intra- and interpersonal movements to become temporally coupled, known as entrainment, induces synchronization for cyclic movements in people walking jointly, swinging pendulums, or rocking chairs alongside each other (Richardson et al. 2005, 2007; Schmidt et al. 1998; van Ulzen et al. 2008). Second, motor simulation allows people to predict a partner's actions based on internal predictive models in their own motor system (Keller et al. 2007; Knoblich and Jordan 2003; Ramnani and Miall 2004; Sebanz and Knoblich 2009; Wolpert et al. 2003).

Most of the studies on entrainment and motor simulation employed continuous rhythmical tasks in which coordination between two people occurred based on visual (Richardson et al. 2005, 2007), haptic (van der Wel et al. 2011), or auditory information (Keller et al. 2007; Konvalinka et al. 2010; Loehr and Palmer submitted). Little is known about how people manage to coordinate their actions at specific points in time in non-rhythmic tasks where continuous feedback about another person is not available. For instance, during a counterattack in football, the attacker often needs to run toward the opponents' goal 
without perceiving what the other players are doing behind his or her back. Such situations require particular processes that allow actors to plan their actions (e.g., run toward a particular position next to the opponents' goal) without exactly knowing when and how the other is going to act (e.g., when the other is going to shoot the ball and where it will land). Thus, coordinating the timing of non-rhythmic, discrete events without continuous information exchange may require the use of coordination strategies, given that all an actor can do is to individually act in a way that will maximize the chances for achieving successful coordination (Vesper et al. 2010).

A powerful coordination strategy is to be as predictable as possible because it allows individuals to rely on and build up common ground (Clark 1996; Brennan and Clark 1996). So far, evidence that people try to be predictable comes mostly from coordination tasks that do not require close temporal coordination. In "Schelling games" (Clark 1996; Schelling 1960), individuals are instructed to coordinate their choices without being able to communicate. In such tasks, people try to be predictable by choosing the most salient option given what they consider to be their common knowledge (Clark 1996). For instance, when two people are asked to pick a meeting point in New York City, they often manage to converge on the same choice, such as Grand Central Station. Moreover, alignment occurring at different levels of speech during conversations is a powerful mechanism of achieving structural and semantic predictability between speaker and listener (Brennan and Clark 1996; Brennan and Hanna 2009; Garrod and Pickering 2004, 2009).

In the present study, we investigated whether coactors also use predictability as a coordination strategy when performing joint tasks that require close temporal coordination in the absence of continuous feedback. Identifying coordination strategies contributes to the understanding of mechanisms involved in real-time coordination beyond entrainment and motor simulation processes.

Focusing on two paradigmatic forms of coordination (Clark 1996), we asked pairs of participants to coordinate their responses to discrete visual events under two sets of instructions. First, to capture "balanced" joint actions where actors have the same roles and equal influence on coordination, we instructed participants to act synchronously. Second, to capture "unbalanced" joint actions involving a leader and a follower, we instructed participants to act in close temporal succession. We predicted that in both tasks people would make use of coordination strategies because the task calls for planning one's own actions without having reliable information about when one's partner is going to act. The time window for coordination in our task was so narrow that acting by the time the partner was seen acting would hardly lead to adequate coordination performance.
If predictability is used as a coordination strategy to solve temporal coordination problems, we should find that participants act as consistently as possible to provide reliable input for each other and by doing so create "procedural" common ground (Clark 1996). This coordination strategy would be reflected in reduced variability of reaction times (RTs) in jointly performed tasks compared with an individual baseline and in correlations between variability and coordination performance. Given that performing tasks at higher speed tends to reduce temporal variability (Repp 2005; Wagenmakers and Brown 2007), we also expected that actions performed in the service of interpersonal coordination would be performed faster to increase predictability.

Furthermore, we aimed at varying coordination difficulty by assigning either the same or opposite stimulus-response (S-R) mappings in a spatial congruency task to pairs of participants. If participants perform the task based on different S-R mappings, coordination difficulty should be greater than when they both act according to the same S-R mapping.

Experiment 1 investigated coordination of actions to be performed synchronously by two coactors. Experiment 2 served to replicate the results of Experiment 1 and addressed the mechanisms underlying the observed effects in more detail. Experiment 3 sought to extend the findings from the synchronous coordination case to sequential coordination, where coactors needed to act in close temporal succession.

\section{Experiment 1}

In Experiment 1, we examined coordination in a joint synchronization task. Both members of a pair performed an individual reaction time task responding to visual stimuli (Simon task; Simon 1990). In the individual condition, participants performed this task alone. In the joint condition, they performed the task next to another participant with the additional constraint to respond at the exact same time as the partner.

We used a spatial congruency task (Simon task) to manipulate how difficult it would be to coordinate one's own responses with those of the partner. The Simon task is a two-choice speeded response task where a task-irrelevant spatial dimension of the stimulus reliably affects individual task performance. In particular, participants react faster to stimuli if they appear on the same side as the required response, e.g., left response to a stimulus on the left (congruent S-R relation; Fig. 1 upper left panel), than when they appear on the opposite side from the required response, e.g., right response to a stimulus on the left (incongruent S-R relation; Fig. 1 lower left panel). The standard explanation is that, although, the spatial position of the stimulus is task-irrelevant, it automatically activates 


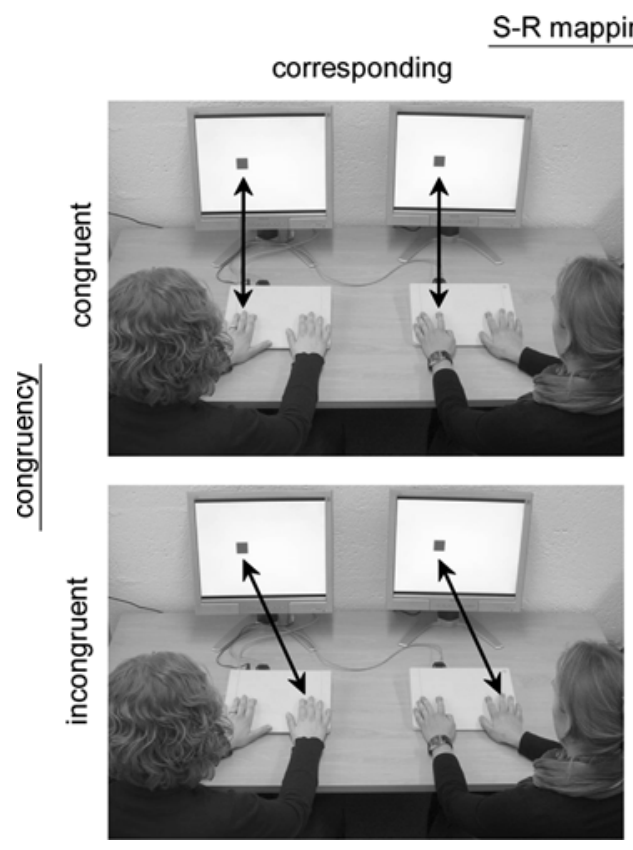

Fig. 1 Setup and design. Pairs of participants performed a two-choice version of the Simon tasks next to each other, responding to the color of visual stimuli on the left or right side of the screen with a left or right button press. The mapping of stimulus and response side was manipulated as a between-subject factor. In the corresponding mapping

the corresponding response because there is an overlap in spatial features (Kornblum et al. 1990).

Both participants in a pair performed the full Simon task by responding with a left or right key press in every trial. By varying whether coactors performed the Simon task with the same or a different S-R mapping, we manipulated how difficult it was to coordinate responses. In one experimental group, the mapping was the same for both members of a pair. Thus, a stimulus that required a congruent response from one participant also required a congruent response from the other participant (corresponding mapping group; Fig. 1 left panels). In the other experimental group, the mapping differed between the two participants, such that a stimulus requiring a congruent response from one participant required an incongruent response from the other participant (non-corresponding mapping group; Fig. 1 right panels). This manipulation posed an extra coordination difficulty for the non-corresponding mapping group compared with the corresponding mapping group because the irrelevant spatial dimension should have the opposite influence on the coactors' response times, thereby making their actions harder to synchronize.

We predicted that participants' RTs would be less variable in the joint condition compared with the individual condition, reflecting the coordination strategy to be as predictable as possible to one's partner. Given that performing tasks at higher speed reduces temporal variability (Repp 2005; Wagenmakers and Brown 2007), we also expected

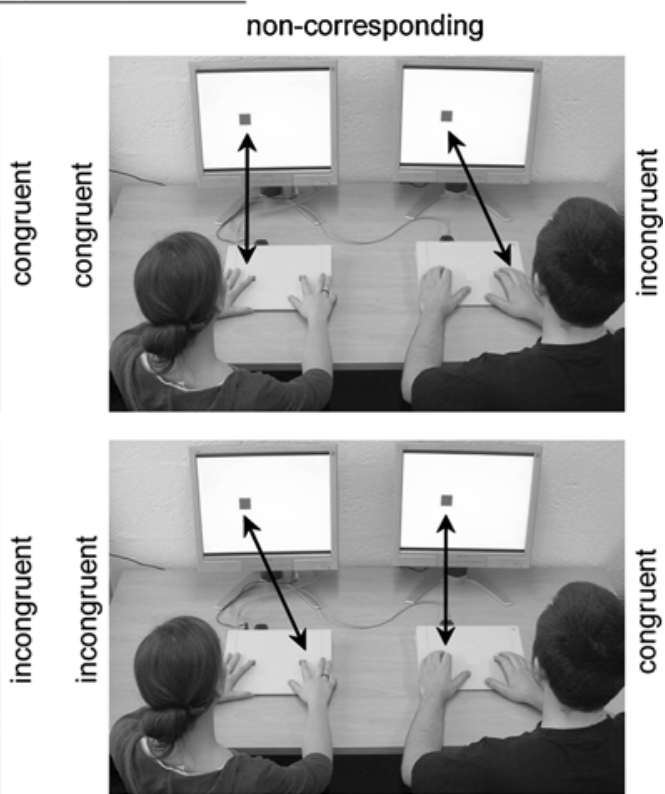

group (left panels), stimuli were always congruent (upper left panel) or incongruent (lower left panel) for both coactors. In the non-corresponding mapping group (right panels), coactors had different mappings such that the same stimulus was at the same time congruent for one participant and incongruent for the other participant

that RTs in the joint condition would be shorter than in the individual condition. Moreover, we expected that reduced variability would have a positive influence on coordination performance, with pairs whose members acted more consistently being better coordinated. Thus, we predicted positive correlations between standard deviation of RTs, mean RTs, and response asynchronies.

In line with previous findings on spatial congruency, we expected RTs to be shorter for congruent trials compared with incongruent trials. Note that finding a congruency effect is a precondition for examining whether having the same or a different S-R mapping affected coordination. To the extent that two participants in a pair show a congruency effect, having non-corresponding S-R mappings should make coordination more difficult than having corresponding $\mathrm{S}-\mathrm{R}$ mappings. Accordingly, the asynchrony between the coactors' RTs should be larger in the non-corresponding group. Alternatively, people may be able to compensate for the additional coordination difficulty by exploiting the coordination strategy of being predictable.

Method

\section{Participants}

Forty undergraduate students (27 women) from Rutgers University Newark, USA, participated for course credit or 
received payment. They were between 17 and 40 years old (mean: 22.1 years). Three participants were left-handed. All reported to have normal or corrected-to-normal vision and gave prior informed consent. Ten pairs of participants were assigned to the corresponding mapping condition and ten to the non-corresponding mapping condition.

\section{Material and apparatus}

The visual stimuli were red and green squares with a border length of $3.0^{\circ}$ visual angle. Each stimulus was shown either on the left or on the right side of the computer screen on a horizontal axis with a distance of $3.0^{\circ}$ visual angle measured from the center. Participants were seated at a distance of approximately $70 \mathrm{~cm}$ from the screen (Fig. 1).

The stimuli were presented with a Mac OS 9.2 computer on either one (individual condition) or two (joint condition) Mitsubishi 17"-screens (resolution $800 \times 600$ pixels; screen refresh rate $120 \mathrm{~Hz}$ ). In the individual condition, each participant sat alone in front of a screen. In the joint condition, two participants sat next to each other and each had their own computer screen. We used PsyScope version 1.2.5 for stimulus presentation and two extension plates of a PsyScope button box to record responses. The data were analyzed with SPSS 15.

\section{Procedure}

Participants received verbal and written instructions. They then performed the individual part of the experiments separately, followed by the joint part. The order was fixed in this first experiment in order to avoid asymmetrical transfer effects between joint and individual performance on the same task that were observed in previous studies (e.g., Atmaca et al. 2008, 2011; Milanese et al. 2010). Each condition consisted of four blocks of 100 trials. The stimuli appeared in random order and with equal frequency. The initial mapping of stimuli to response keys was counterbalanced and changed after two blocks. For each participant, the initial mapping in the individual condition was the same as the initial mapping in the joint condition.

The individual task started with a practice session of 24 trials, after which the experimental run started. Each trial began with a fixation cross presented for $500 \mathrm{~ms}$ followed by the stimulus that was shown for $1,000 \mathrm{~ms}$, or until a button was pressed. We used a 1,000 ms intertrial interval. Participants were instructed to respond as quickly and as accurately as possible to the color of the stimulus by pressing one of the two buttons (left or right) on a button box with their left or right index finger. Participants received auditory error feedback through a short tone presented immediately after an incorrect button press. A second error feedback (a different tone) signaled to the participants that they were too slow (RT $>800 \mathrm{~ms}$ ). The joint condition also started with a practice session of 24 trials and was the same as the individual condition except that the two members of a pair were instructed to react as synchronously as possible ("try to respond at exactly the same time").

Results

We conducted mixed ANOVAs with the within-subject factors Condition (individual condition vs. joint condition) and Congruency (congruent vs. incongruent) and the between-subject factor Correspondence (corresponding mapping vs. non-corresponding mapping) on standard deviation of reaction time (STD) and mean reaction time (RT). In addition, we computed partial and zero-order correlations between STD, RTs, and the asynchrony between the two coactors' RTs to determine the relative contributions of RTs and variability to successful performance. Error trials (wrong key, no response, or response not in a time window of $200-1,000 \mathrm{~ms}$ ) were removed from the analyses and are reported in more detail later. We report the analyses in the order following our predictions, that is, we first report the main effects of Condition from the ANOVAs on STD and $\mathrm{RT}$, followed by the correlation analyses, and we last report the remaining results from the ANOVAs pertaining to Congruency and Correspondence. To ensure that any differences between individual and joint conditions were not due to simple training effects, two one-way ANOVAs with the within-subject factor Block (1-4) were performed on the RT data to assess changes in performance speed (Fig. 2b). They revealed no significant changes in the individual condition and in the joint condition, all $P>.06$. Therefore, the data were pooled over blocks for the remaining analyses.

\section{Variability and speed}

Consistent with our predictions, RTs were less variable in the joint condition, as shown by a main effect of Condition in the ANOVA performed on STD, $F(1,38)=86.2$, $P<.001$ (Fig. 2a). RTs were also faster in the joint condition than in the individual condition, $F(1,38)=168.26$, $P<.001$ (Fig. 2b). RT and STD were significantly correlated, as shown by zero-order correlations (Fig. 2c). The faster the responses, the less variable they tended to be. Both RT and STD were also significantly correlated with asynchrony (mean asynchrony: $73.2 \mathrm{~ms}$ ). Thus, coordination performance of a pair was better the faster and less variable participants' responses were. To determine the relative contribution of RT and STD for asynchrony, partial correlations were performed. The analyses showed that when controlling for RT, STD was still significantly correlated with asynchrony. However, RTs were not significantly correlated with asynchrony when controlling for 

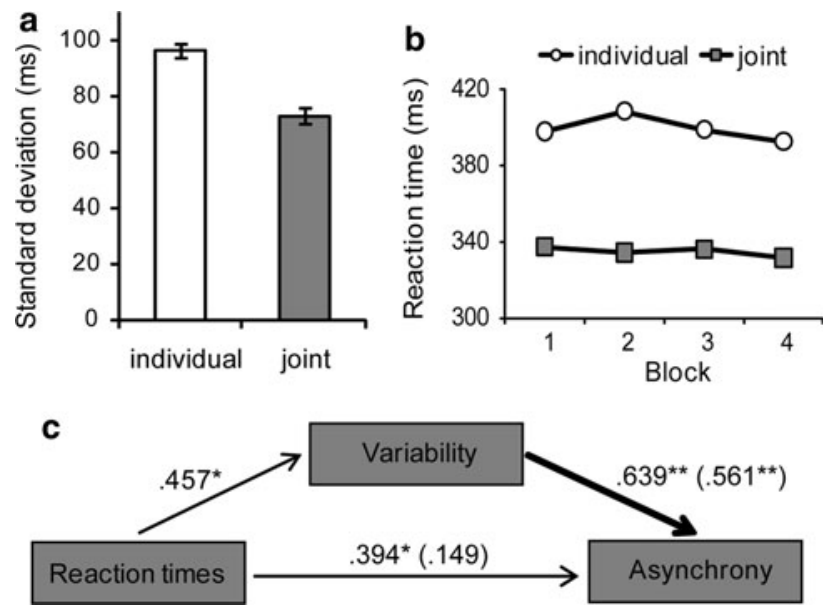

Fig. 2 Results of Experiment 1 (synchronous coordination). a Reaction times were less variable in the joint condition than in the individual condition. Error bars display within-subject confidence intervals (Loftus and Masson 1994). b Reaction times were faster in the joint compared with the individual condition. $\mathbf{c}$ Zero-order correlations showed that response asynchronies were positively correlated with standard deviation and mean reaction times. Moreover, partial correlations (in brackets) suggest that variability had a direct influence on asynchrony (thick black arrow), whereas speeding supported coordination only indirectly (significance levels: $* P<.05 ; * * P<.001$ )

STD. This suggests that the reduction in variability directly contributed to coordination performance.

\section{Coordination difficulty}

As expected, there was a spatial congruency effect both in the individual and in the joint condition, $F(1,38)=97.53$, $P<.001$. RTs were overall faster in congruent trials (stimulus and response side the same) than in incongruent trials (stimulus and response side different).

However, despite the fact that participants showed a significant congruency effect, asynchrony between participants' responses in the joint condition did not differ significantly between the corresponding $(73.6 \mathrm{~ms})$ and the non-corresponding $(72.9 \mathrm{~ms})$ group, $t(38)=.23$, $P>.8$. The mixed ANOVA performed on STD showed a significant main effect of Congruency, $F(1,38)=14.4$, $P<.01$, but no main effect of Correspondence, $F(1,38)=.67, P>.4$. There was no significant interaction between Condition and Correspondence, $F(1,38)=.01, P>.9$. None of the other interactions were significant, except for the interaction between Condition and Congruency, $F(1,38)=7.03, P<.05$. The same ANOVA performed on RT showed neither a main effect of Correspondence, $F(1,38)=3.4, P>.07$, nor a significant interaction between Condition and Correspondence, $F(1,38)=.04, P>.8$. The other interactions were also not significant.

\section{Errors}

The same mixed ANOVA was performed on errors. Overall, participants made more errors in the joint condition $(12.4 \%)$ than in the individual condition (5.2\%), $F(1,38)=46.38, P<.001$. To test whether the increased error rate in the joint condition is due to the speeding up of RTs, we correlated the mean amount of errors with mean RTs. The faster the RTs the more errors were committed, $r=-.41, P<.01$. There was also a significant main effect of Congruency, $F(1,38)=19.18, P<.01$, with more errors occurring on incongruent trials. The main effect of Correspondence was not significant, $F(1,38)=0.45, P>.5$. None of the interactions were significant, apart from the interaction between Condition and Congruency, $F(1,38)=18.45$, $P<.001$. The increase in errors on incongruent trials compared with congruent trials was more pronounced in the joint condition.

\section{Discussion}

The findings confirmed our hypothesis that people use predictability as a coordination strategy to achieve synchronization. RTs were less variable in the joint condition compared with the individual condition. Predictability was likely achieved through a speed up of RTs, as suggested by positive correlations between RT and STD. This is in line with previous findings showing that performing tasks at higher speed reduces temporal variability (Repp 2005; Wagenmakers and Brown 2007). Importantly, partial correlations showed that the reduction in variability was key in reducing the asynchrony between coactors' responses. The reduction in RTs (i.e., the speeding up) only contributed indirectly to coordination by reducing variability. Speeding up also resulted in a higher error rate in the joint condition which likely reflects a criterion shift toward speed at the cost of accuracy. Participants may have accepted higher error rates given that speeding up reduced variability.

In line with previous findings on spatial congruency, we found shorter RTs and lower error rates on congruent trials compared with incongruent trials. ${ }^{1}$ The presence of a congruency effect in RTs was a precondition for examining whether having the same or a different S-R mapping affected how well participants were coordinated. To the extent that two participants in a pair show a congruency effect, having non-corresponding $\mathrm{S}-\mathrm{R}$ mappings should pose more coordination difficulties than having corresponding S-R mappings. Accordingly, we expected the asynchrony between the coactors' RTs to be larger in the

\footnotetext{
${ }^{1}$ The interaction between Condition and Congruency (higher error rate for incongruent trials in the joint condition compared to the individual condition) is likely due to the shorter RTs in the joint condition.
} 
non-corresponding group. However, the comparison between the corresponding and the non-corresponding mapping group showed that pairs achieved approximately equal degrees of synchronization regardless of mapping correspondence. We also did not find evidence to suggest that participants compensated for the additional coordination difficulty by decreasing variability even more in the non-corresponding mapping group. We conclude from this pattern that, most likely, the manipulation of coordination difficulty was not sensitive enough to reveal changes in joint task performance. Given that the mean asynchrony was substantially larger (around $70 \mathrm{~ms}$ ) than the average congruency effect (around $20 \mathrm{~ms}$ ), assigning different S-R mappings to coactors may not have increased coordination difficulty to a sufficient degree.

\section{Experiment 2}

The aim of Experiment 2 was to replicate the results of Experiment 1 and to address the mechanisms underlying the observed effects in more detail. One could argue that the reduction in variability in the joint condition observed in Experiment 1 does not reflect a coordination strategy but is simply a consequence of performing the task next to another person (social facilitation; Aiello and Douthitt 2001; Zajonc 1965). In particular, it is known that people performing tasks next to each other tend to fall into synchrony even when not intending to do so (Richardson et al. 2005, 2007). To test whether intending to synchronize is a necessary precondition for the relation between reduced variability and asynchrony to emerge, we compared a condition in which participants performed independent RT tasks without intending to coordinate (joint unintentional condition) to a condition in which they tried to synchronize their responses in the same way as in Experiment 1 (joint intentional condition). In all other respects, the experiment was identical to Experiment 1. If the intention to synchronize was critical, we should find that a reduction in variability reduces asynchrony in the joint intentional condition. This would suggest that making oneself predictable constitutes a coordination strategy. Otherwise, we should find the same pattern in both the joint intentional and the joint unintentional condition.

In contrast to Experiment 1, we counterbalanced the order of conditions. This allowed us to also assess possible transfer effects between joint and individual task performance (Atmaca et al. 2008, 2011; Milanese et al. 2010). Moreover, to ensure that the results of Experiment 2 could be compared with Experiment 1, we again varied whether the two participants in a pair had corresponding or non-corresponding $\mathrm{S}-\mathrm{R}$ mappings.
Method

\section{Participants}

Forty undergraduate students (27 women) from Radboud University Nijmegen, the Netherlands, participated for course credit or received payment. They were between 18 and 35 years old (mean: 23.4 years). Three participants were left-handed. All reported to have normal or correctedto-normal vision and gave prior informed consent. Ten pairs of participants were assigned to the corresponding mapping group and another ten to the non-corresponding mapping group.

\section{Material and apparatus}

We used the same stimuli as in Experiment 1. Stimulus presentation and data collection were performed with the experimental software Presentation on a Hewlett Packard PC (Windows Vista). Stimuli were displayed on either one (individual condition) or two (joint intentional and unintentional conditions) Philips 19"'-screens (resolution of $1,280 \times 1,024$ pixels, screen refresh rate $60 \mathrm{~Hz}$ ). Responses were recorded with two Presentation response boxes. We analyzed the data with SPSS 15.

\section{Procedure}

The procedure was the same as in Experiment 1 with the following exceptions: First, in addition to the individual and joint condition, we added a "joint unintentional condition" in which participants performed independent tasks next to each other. Apart from the instructions that were, as in the individual condition, to respond as fast and as accurately as possible, this condition was identical to the other joint condition ("joint intentional"). Second, there were only 200 trials in each condition to avoid effects of fatigue. Third, the order of the three conditions was counterbalanced with respect to individual and joint performance. Half of the participant pairs started with the individual condition and then performed the two joint conditions. The other half started with the two joint conditions and then performed the individual condition. The joint unintentional condition was always performed before the joint intentional condition to avoid transfer effects, such that participants would maintain the intention to synchronize their responses with the coactor in the joint unintentional condition after having performed the joint intentional condition.

\section{Results}

As in Experiment 1, we conducted mixed ANOVAs with the within-subject factors Condition (individual condition 
vs. joint intentional condition vs. joint unintentional condition) and Congruency (congruent vs. incongruent) and the between-subject factor Correspondence (corresponding mapping vs. non-corresponding mapping) on standard deviation of reaction time (STD) and mean reaction time (RT). Bonferroni-corrected comparisons were used to compare the three conditions. In addition, we computed partial and zero-order correlations between STD, RTs, and the asynchrony of the two coactors' RTs. Error trials (wrong key, no response, or response not in time window of 2001,000 $\mathrm{ms}$ ) were removed from the analyses and are reported in more detail later. As in Experiment 1, we first analyzed the RT data blockwise to ensure that any differences between conditions were not due to simple training effects (Fig. 3b). Paired-samples t-tests revealed no significant changes from block 1 to block 2 in the joint intentional and the joint unintentional conditions, all $P>.7$. In the individual condition, participants reacted more slowly in the second block than in the first block, $t(39)=-2.34, P<.05$, which is opposite to what would be expected by a practice effect. Therefore, the data were pooled over blocks for the remaining analyses.

\section{Asynchrony}

Participants' responses were significantly more synchronized in the joint intentional condition (mean asynchrony $69.6 \mathrm{~ms}$ ) compared with the joint unintentional condition (mean asynchrony $78.1 \mathrm{~ms}), t(39)=-3.51, P<.01$.

\section{Variability and speed}

The ANOVA on STD showed a significant main effect of Condition, $F(2,76)=8.46, P<.001$ (Fig. 3a). Responses in the joint intentional condition and in the joint unintentional condition did not differ from each other in terms of STD $(P>.6)$, but responses in both conditions were significantly less variable than responses in the individual condition, all $P<.01$

With respect to RT, there were no significant differences between conditions (all $P>.1$; Fig. 3b). However, as in the joint condition of Experiment 1, RT and STD in the joint intentional condition were significantly correlated (Fig. 3c). The faster the responses, the less variable they tended to be. Both RT and STD were also significantly correlated with asynchrony. Thus, coordination performance of a pair was better the faster and less variable participants' responses were. To determine the relative contribution of RT and STD for asynchrony, partial correlations were calculated. As in Experiment 1, the analyses showed that when controlling for RT, STD was still significantly correlated with asynchrony. However, RTs were not significantly correlated with asynchrony when controlling for STD. This
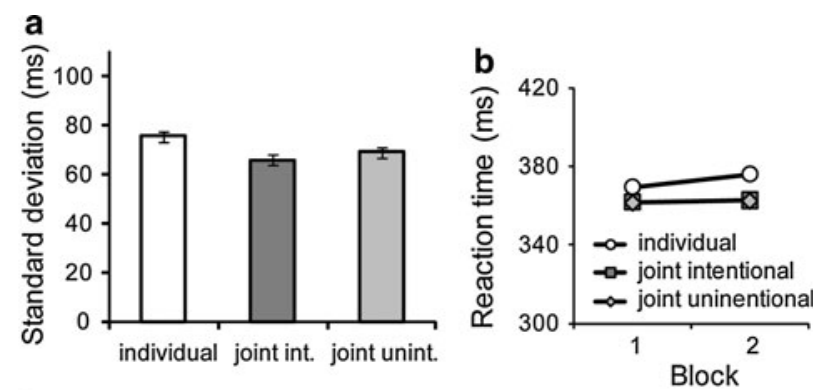

C

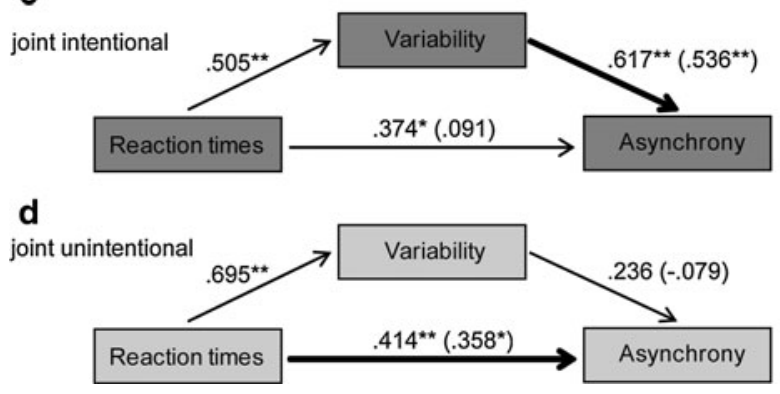

Fig. 3 Results of Experiment 2 (synchronous coordination). a Reaction times were less variable in the joint intentional condition compared with the individual condition. Mean standard deviation was also reduced in the joint unintentional condition. Error bars display within-subject confidence intervals (Loftus and Masson 1994). b Reaction times were not faster in the joint intentional and unintentional conditions than in the individual condition. $\mathbf{c}$ In the joint intentional condition, response asynchronies were positively correlated with standard deviation and mean reaction times as shown by zero-order correlations. Partial correlations (in brackets) support the prediction that only response variability had a direct influence on asynchronies (thick black arrow), whereas speeding supported coordination indirectly (significance levels: $* P<.05 ; * * P<.001$ ). c In the joint unintentional condition, only speed was correlated with asynchronies (thick black arrow) as shown by zero-order and partial correlations (in brackets). Variability of responses did not influence how well coactors were coordinated

confirms that the reduction in variability directly contributed to coordination performance. In the joint unintentional condition, RT was significantly correlated with STD and asynchrony (Fig. 3d). Importantly, however, in contrast to the joint intentional condition, STD and asynchrony were not significantly correlated in the joint unintentional condition.

\section{Order}

As reported previously, the RT results differ from Experiment 1 in that mean RT was not faster in the joint intentional condition compared with the individual condition. Given that the only difference between Experiments 1 and 2 was the introduction of a third condition (joint unintentional), we investigated whether the order in which the conditions had been performed affected RTs. A within-subject ANOVA with the factors Condition (individual vs. joint intentional vs. joint unintentional) and Order (joint conditions 
Fig. 4 Effect of the order of conditions in Experiment 2. a Reduced variability in the joint intentional and joint unintentional conditions was found when participants performed the individual condition first. b Responses were also faster in the joint conditions in participants starting with the individual condition. c Variability was not reduced when participants performed the joint condition first. d Also, the reaction times were not reduced in participants starting with the joint condition. Error bars display within-subject confidence intervals (Loftus and Masson 1994)
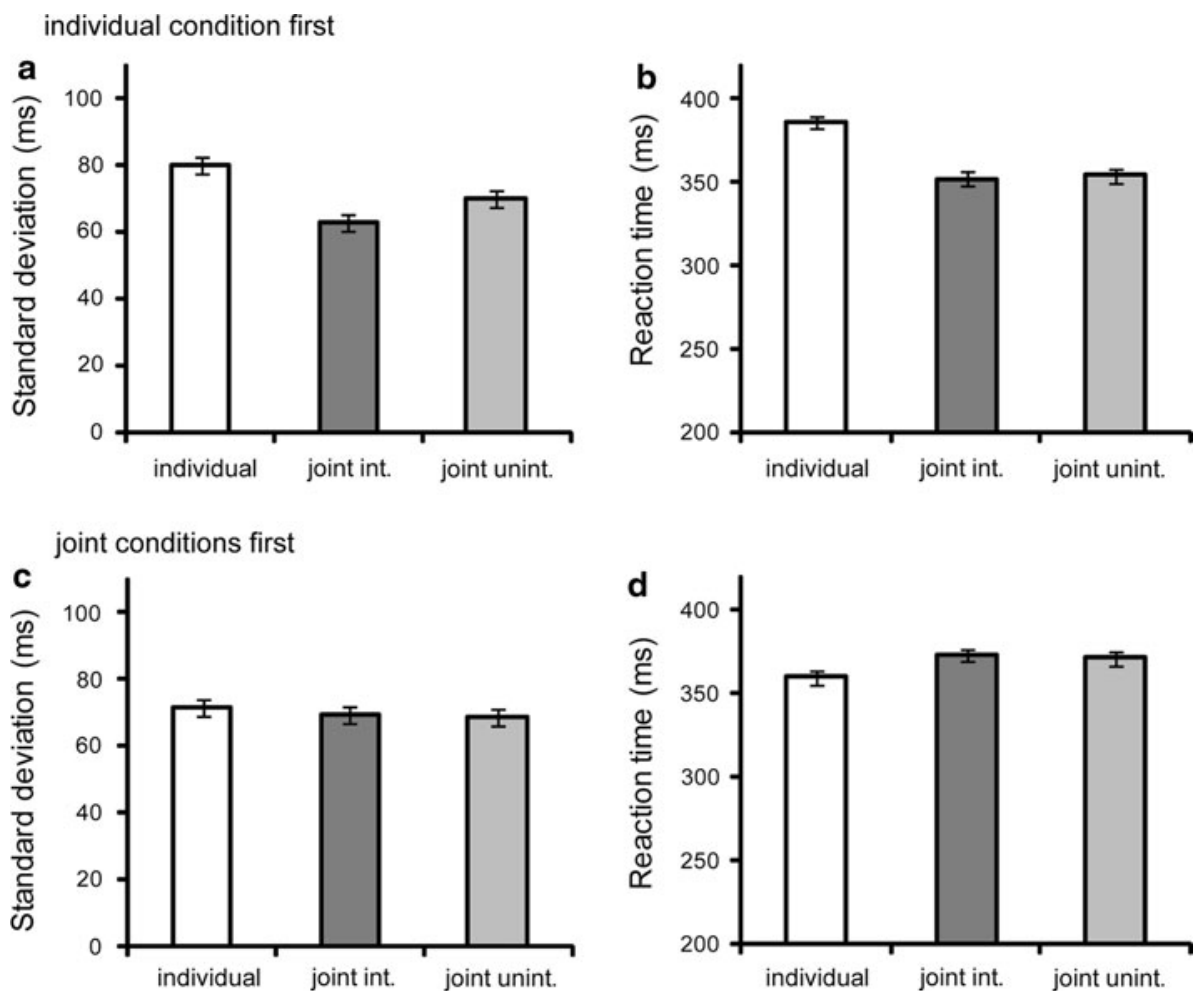

performed first vs. individual condition performed first) showed not only a significant main effect of Condition, $F(2,76)=4.9, P<.05$, but also a significant interaction between Condition and Order, $F(2,76)=23.5, P<.001$. For participants who performed the individual condition first, RTs were significantly faster in the joint intentional condition than in the individual condition, $t(19)=5.07$, $P<.001$ (Fig. 4b), replicating the pattern observed in Experiment 1. RTs in the joint unintentional condition were also faster than RTs in the individual condition, $t(19)=5.39, P<.001$. However, participants who performed the joint conditions first showed the opposite pattern, RTs being faster in the individual condition than in the joint intentional condition, $t(19)=-2.14, P<.05$, and the joint unintentional condition, $t(19)=-2.6, \quad P<.05$ (Fig. 4d).

In the next step, we performed the same ANOVA on STD to explore whether the reduction in STD in the joint conditions was more pronounced in participants who performed the individual condition first. In addition to a significant main effect of Condition, $F(2,76)=9.57, P<.001$, there was indeed a significant interaction between Condition and Order, $F(2,76)=5.47, P<.01$. Participants who performed the individual condition first showed a significant reduction in STD in the joint intentional condition compared with the individual condition, $t(19)=4.45$, $P<.001$, as well as in the joint unintentional condition, $t(19)=3.32, P<.01$ (Fig. 4a). In contrast, STD in partici- pants who performed the joint conditions first did not differ across conditions (all $P>.1$; Fig. 4c).

Finally, Order had no critical effect on the pattern observed in the correlation analyses. In the joint intentional condition, the partial correlation between STD and asynchrony was significant when controlling for RT regardless of order (individual first: $r=.46, P<.05$; joint first: $r=.59$, $P<.01)$. In the joint unintentional condition, this correlation was not significant, regardless of order (individual first: $r=.032, P>.8$; joint first: $r=-.31, P>.1$ ).

\section{Coordination difficulty}

There was a significant main effect of Congruency, $F(1,38)=75.85, P<.001$. RTs were overall faster in congruent trials (stimulus and response side the same) than in incongruent trials (stimulus and response side different). Based on this finding, we examined whether in this experiment the between-group factor Correspondence had an influence on RT. Despite the fact that participants showed a significant congruency effect, asynchrony between participants' responses in the joint intentional condition did not differ significantly between the corresponding $(70.5 \mathrm{~ms})$ and the non-corresponding $(68.7 \mathrm{~ms})$ group, $t(38)=.45$, $P>$.6. The same was true for the joint unintentional condition, $t(38)=-.14, P>.8$ (corresponding: $77.7 \mathrm{~ms}$; noncorresponding: $78.5 \mathrm{~ms}$ ). The mixed ANOVAs performed on STD and RT did not reveal any significant effects apart 
from the main effect of Congruency (STD: $F(1,38)=5.89$, $P<.05$; RT: $F(1,38)=79.85, P<.001)$.

\section{Errors}

The same mixed ANOVA as on STD and RT was performed on errors and showed a significant main effect of Condition, $F(2,76)=4.6, P<.05$. The difference in error rates between the individual condition $(2.8 \%)$ and the joint intentional condition $(4.1 \%)$ was close to significant $(P=.05)$. The joint unintentional condition $(3.5 \%)$ was not significantly different from either of the other conditions. Mean RTs in the joint intentional condition were negatively but not significantly correlated with the error rate $(r=-.19$, $P>$.2). There was a significant main effect of Congruency, $F(1,38)=13.45, P<.01$, with more errors occurring on incongruent trials. The main effect of Correspondence was not significant, $F(1,38)=2.98, P>.07$. None of the interactions were significant.

\section{Discussion}

The results of Experiment 2 confirm the relation between STD, RT, and asynchrony observed in Experiment 1 . In the joint intentional condition where participants were instructed to synchronize their responses, RT and STD were significantly correlated with each other and with the size of the asynchrony. Coordination performance of a pair was better the faster and less variable participants responses were. As in Experiment 1, the partial correlations showed that when controlling for RT, STD was still significantly correlated with asynchrony whereas RT was not significantly correlated with asynchrony when controlling for STD. This provides further evidence for the claim that a reduction in variability directly contributes to synchronization.

To address the concern that the reduction in variability in the joint condition may be a consequence of performing the task next to another person, we included the joint unintentional condition where participants performed the same task next to each other without intending to synchronize. The results showed that although RT and STD were correlated, STD did not influence asynchrony. This provides clear evidence for the claim that the reduction in variability constitutes a coordination strategy and does not simply occur when people act together without the intention to coordinate as in cases of social facilitation where people generally speed up when others are present (Aiello and Douthitt 2001; Zajonc 1965). By reducing variability, coactors become more predictable to each other, which may help to build up procedural common ground (Clark 1996).

As in Experiment 1, the reduction in variability in the joint intentional condition was achieved at least partly by speeding up. The speeding up of RTs was less clear than in
Experiment 1, because we only found a significant difference between the individual and the joint intentional condition when the individual condition was performed before the joint conditions. Most likely, we did not find shorter RTs in the joint conditions throughout due to a carryover effect; when participants performed the joint conditions first, the focus on speed may have carried over to the individual condition performed subsequently.

Interestingly, the correlation analyses of the joint unintentional condition suggest that faster responses can also directly contribute to coordination. This indicates that speeding per se may lead to synchronization even when coactors do not intend to be coordinated (Vesper et al. 2010). As in Experiment 1, coordination difficulty, as manipulated by assigning corresponding or non-corresponding S-R mappings to participants in a pair, did not affect performance.

\section{Experiment 3}

The aim of Experiment 3 was to test whether the relation between RT, STD, and coordination performance generalizes to other coordination tasks. In Experiment 1 and 2, participants were instructed to act synchronously to capture "balanced" joint actions where actors have the same roles and equal influence on coordination. According to Clark (1996), another paradigmatic form of coordination is given in "unbalanced" joint actions involving a leader and a follower. To address this form of coordination, we instructed participants in Experiment 3 to act as quickly as possible but in a particular order.

Pairs of participants performing the Simon task were instructed to respond shortly after one another in a prespecified order. This sequential task constraint implied a clear assignment of roles to participants as they acted either as leader or follower. Due to this role differentiation, sequential joint coordination is different from synchronous coordination. However, the leader faces similar challenges as coactors in the synchronous coordination task because all the leader can do to maximize the chances of successful coordination is to act as predictably as possible. Therefore, we expected leaders to make use of the same coordination strategy as coactors in Experiment 1 and 2. The time window during which both responses had to be given in succession was so narrow that adequate coordination performance could only be achieved if the follower predicted the timing of the leader's actions. We expected that the leader, by reducing variability of her responses, might be able to support the follower in timing her actions so that they occur after the leader's actions with a minimal delay. There was no joint unintentional condition because such a condition would not provide a useful comparison to the sequential 
coordination task where coactors intentionally perform actions in close temporal succession.

As in Experiment 1 and 2, we varied whether the two participants in a pair had corresponding or non-corresponding S-R mappings to investigate whether coordination difficulty differentially affects balanced and unbalanced coordination tasks.

\section{Method}

\section{Participants}

Forty students (24 women) of the University of Munich, Germany, aged between 19 and 32 years (mean: 23.8 years) participated in the experiment. They were paid for their participation. All participants had normal or corrected-to-normal vision, and two were left-handed. Ten pairs of participants were assigned to the corresponding mapping group and ten pairs to the non-corresponding mapping group.

\section{Material and apparatus}

We used the same stimuli as in Experiment 1 and 2. Stimulus presentation and data collection were performed with an Apple Power PC. Stimuli were displayed on either one (individual condition) or two (joint condition) Apple 21"'screens (resolution of $1,024 \times 768$ pixels, screen refresh rate $120 \mathrm{~Hz}$ ). Responses were recorded with the same extension plates of a PsyScope button box as in Experiment 1. We analyzed the data with SPSS 15 for Windows.

\section{Procedure}

Half of the participant pairs started with the individual condition, while the other half performed the joint condition first. Before each condition, verbal and written task instructions were given. The individual condition consisted of three blocks of 100 trials each and the joint condition of six blocks of 100 trials each. The stimuli appeared in random order and with equal frequency. The mapping of stimulus color to response location was counterbalanced and identical for a given participant's individual and joint condition.

The individual condition started with 60 practice trials, followed by the experimental run. All trials had the same structure as trials in Experiment 1 and 2. The joint condition was also preceded by 60 practice trials. In addition to the instruction to respond as quickly and as accurately as possible, participants in the joint condition were instructed to respond to the stimuli in a specific order ("try to respond after the other as quickly as possible"). The response order was determined by the stimulus color. For example, the person seated on the left side responded first when a red stimulus appeared, and the person seated on the right side responded first when a green stimulus appeared. This mapping of response order and stimulus color was counterbalanced between pairs and changed for each pair after the third joint block.

Results

Leaders' performance was analyzed with mixed ANOVAs with the within-subject factors Condition (individual condition vs. joint condition) and Congruency (congruent vs. incongruent) and the between-subject factor Correspondence (corresponding mapping vs. non-corresponding mapping) on standard deviation of reaction time (STD) and mean reaction time (RT). In addition, we computed partial and zero-order correlations between STD, RTs, and the asynchrony between the two coactors' RTs. Followers' responses were only considered in so far as they concerned leaders' performance, i.e., as contributing to the pair's asynchronies. Error trials (wrong key, no response, responses shorter than $200 \mathrm{~ms}$ or longer than 1,000 ms, or responses in which the follower responded at the same time or before the leader) were removed from the analyses and are reported in more detail later. To ensure that differences between conditions were not due to simple learning effects, we first analyzed the RT data blockwise (Fig. 5b). Two one-way ANOVAs with the within-subject factor Block (1-3 for individual, 1-6 in joint) revealed no significant changes in the individual and joint conditions, all $P>.08$. Therefore, the data were pooled over blocks for the remaining analyses.

\section{Variability and speed}

In line with our predictions, RTs were less variable in the joint condition, as shown by a significant main effect of Condition in the ANOVA performed on STD, $F(1,38)=21.24$, $P<.001$ (Fig. 5a). RTs were also faster in the joint condition than in the individual condition, $F(1,38)=15.22, P<.001$ (Fig. 5b). RT and STD were significantly correlated, as shown by zero-order correlations (Fig. 5c), such that responses were less variable the faster they were. Both RT and STD were also significantly correlated with asynchrony (mean asynchrony: $204.3 \mathrm{~ms}$ ). The partial correlation analyses showed that when controlling for RT, STD was still significantly correlated with asynchrony. However, RTs were not significantly correlated with asynchrony when controlling for STD. This confirms that the reduction in variability directly contributed to coordination performance.

\section{Coordination difficulty}

There was a significant main effect of Congruency, $F(1,38)=141.53, P<.001$. Leaders' RTs were overall 

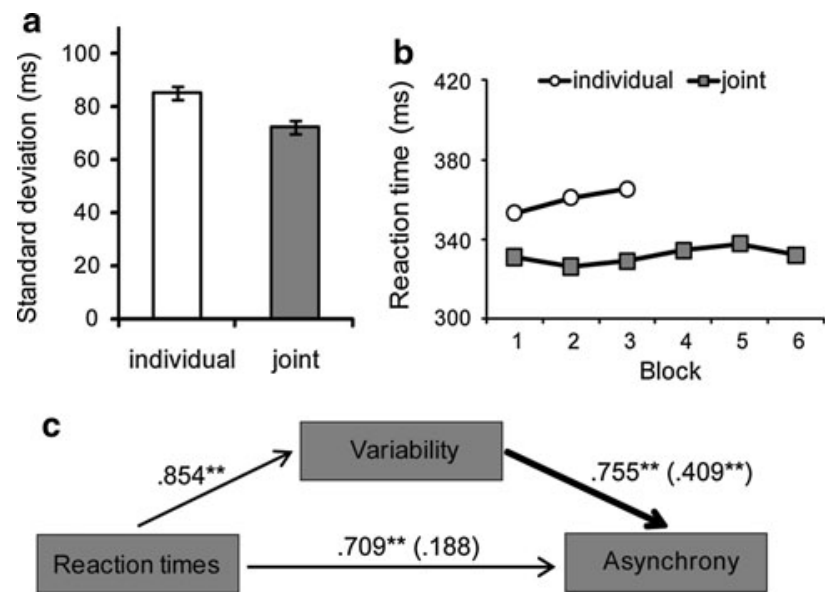

Fig. 5 Results of Experiment 3 (sequential coordination). a Leaders' reaction times were less variable (reduced standard deviation) in the joint condition than in the individual condition. Error bars display within-subject confidence intervals (Loftus and Masson 1994). b Reaction times of the leader were faster in the joint compared with the individual condition. c Zero-order correlations showed that the asynchrony between leaders' and followers' responses was positively correlated with the standard deviation and mean reaction times of the leader. Moreover, partial correlations (in brackets) indicate that the leader's response variability had a direct influence on asynchrony (thick black arrow), whereas speeding supported coordination only indirectly (significance levels: $* P<.05 ; * * P<.001$ )

faster on congruent trials (stimulus and response side the same) than on incongruent trials (stimulus and response side different).

The asynchrony between participants' responses in the joint condition did not differ significantly between the corresponding (195.6 $\mathrm{ms})$ and the non-corresponding (213.1 ms) group, $t(38)=-.8, P>$.4. The mixed ANOVA performed on STD showed a marginally significant main effect of Congruency, $F(1,38)=3.74, P=.06$, but no main effect of Correspondence, $F(1,38)=.31, P>.5$. There was no significant interaction between Condition and Correspondence, $F(1,38)=.01, P>.9$. None of the other interactions were significant, except for the interaction between Condition and Congruency, $F(1,38)=5.58, P<.05$. The same ANOVA performed on RT showed neither a main effect of Correspondence, $F(1,38)=.67, P>.4$, nor any significant interactions.

\section{Errors}

The same mixed ANOVA was performed on errors. The error rate in the individual $(6.1 \%)$ and in the joint condition $(6.9 \%)$ did not differ significantly, $F(1,38)=.62, P>.4$. The main effect of Congruency only showed a tendency, $F(1,38)=3.05, P<.09$, with slightly more errors occurring on incongruent trials. The main effect of Correspondence was not significant, $F(1,38)=0.25, P>.6$. Unlike in the two previous experiments, we found a significant interaction between Correspondence and Congruency, $F(1$, $38)=5.78, P<.05$, and a significant three-way interaction between Condition, Congruency and Correspondence, $F(1,38)=4.75, P<.05$. As in Experiment 1 , there was a significant interaction between Condition and Congruency, $F(1,38)=4.9, \quad P<.05$, but the pattern was reversed (smaller congruency effect in the joint condition).

Discussion

Experiment 3 provides evidence that the relation between RT, STD, and coordination performance generalizes from synchronous to sequential coordination. The faster and less variable the leaders' responses were, the better the coordination performance of coactors responding as quickly as possible after one another. This demonstrates that reducing variability is a powerful coordination strategy across different coordination tasks. As for the synchronous coordination task, partial correlations showed that STD was significantly correlated with asynchrony when controlling for RT. RT, however, was not significantly correlated with asynchrony when controlling for STD. This confirms that also in a sequential coordination task reduced variability directly contributes to better coordination performance, with speeding helping to reduce variability.

This observed generalization from a synchronization task to a sequential task is interesting because it suggests that the same general coordination strategy can be useful in two tasks that pose different challenges and constraints to the collaborating individuals. For example, whereas it is evident that both coactors in the synchronous task contribute to the joint outcome, this is less intuitive in the sequential task because the follower seems to take most of the responsibility for coordination. Still, the asynchronies are determined by both people's response times and, therefore, also depend crucially on the timing of the leader's actions. The current findings show that, although the sequential coordination task differs from the synchronous coordination task in several respects, reducing variability proved to be a useful coordination strategy on the leader's part in the sequential task because it allowed the follower to predict the timing of the leader's actions.

As in the synchronous task, varying the correspondence between coactors' S-R mappings did not affect coordination performance. This is in line with the findings of Experiment 1 and 2 and indicates that subtle differences in coordination difficulty neither impaired performance in the balanced nor in the unbalanced coordination task.

\section{General discussion}

The aim of the present study was to investigate the mechanisms that allow coactors to temporally coordinate the 
onset of discrete actions in a non-rhythmic task. We hypothesized that if continuous feedback about a coactor's performance is not available, coordination strategies will be used to facilitate coordination. Based on prior research on coordination problems showing that coactors try to act in a predictable way to facilitate coordination (Clark 1996; Schelling 1960), we tested whether predictability is also used as a coordination strategy to achieve close temporal coordination.

Across three experiments, we found that coactors reduced the variability of their actions in a joint coordination task compared with the same task performed individually. Importantly, correlation analyses showed that the less variable the actions were, the better the resulting interpersonal coordination, as measured by the asynchrony between participants' responses. This was true both for a synchronization task where pairs of participants were instructed to respond to visual events at the same time (Experiments 1 and 2) and for a sequential coordination task where participants needed to act one after the other in close temporal succession (Experiment 3). The relation between reduced variability and improved coordination performance was not observed when pairs of participants performed independent tasks next to each other without intending to coordinate (Experiment 2). This suggests that the reduction in variability formed part of a coordination strategy.

In addition, the results indicate a link between the speed and the variability of responses. In all three experiments, speed correlated with variability, such that the faster the responses, the less variable they tended to be. In Experiment 1 and 3 , responses were also generally faster when participants acted together compared with when they performed the same task alone. In Experiment 2, this pattern held for participants who performed the individual task before the joint tasks but not for participants who performed the joint intentional and joint unintentional task first. Participants starting with the joint conditions were faster in the subsequently performed individual condition, which likely reflects carryover effects from joint to individual task performance.

What exactly is the role of speeding for coordination? The partial correlation analysis showed that response speed in the joint conditions in Experiment 1 and 3 and in the joint intentional condition in Experiment 2 was not significantly correlated with the asynchrony of coactors' responses when controlling for variability. In contrast, when controlling for response speed, variability was still significantly correlated with asynchrony. This suggests that the reduction in variability directly contributed to coordination performance, whereas speeding played only a mediating role by reducing variability. The pattern observed in the joint unintentional condition where participants performed independent tasks next to each other markedly differs from this; in the joint unintentional condition response speed was significantly correlated with asynchrony even when controlling for variability, whereas variability and asynchrony were not significantly correlated. This indicates that when participants performed independent tasks, their responses became coordinated to some extent (albeit not to the same extent as during intentional coordination) through fast task performance.

The present experiments also attempted to investigate whether joint performance is modulated by how difficult it is to achieve precise temporal coordination. We reasoned that coordination difficulty should be greater when two coactors perform the spatial congruency task with opposite $\mathrm{S}-\mathrm{R}$ mappings compared with when they have the same $\mathrm{S}-\mathrm{R}$ mappings. We expected the asynchrony between the coactors' RTs to be larger in the non-corresponding group where spatially congruent trials for one actor were spatially incongruent for the other actor. Unexpectedly, even though participants showed a spatial congruency effect, asynchrony was not affected by whether or not their S-R mappings corresponded. Probably, the correspondence manipulation was too subtle given that the mean asynchrony between participants' responses was substantially larger than the average congruency effect. It seems that participants were able to compensate for what were probably just subtle differences in coordination difficulty. Stronger manipulations of task difficulty are needed to investigate how coactors compensate for large asynchronies induced by differences in the tasks they perform.

Our main finding that reducing variability aids coordination extends prior research on temporal coordination. So far, research has focused on entrainment and motor simulation as key mechanisms underlying coordination. We believe that the reduction in variability observed in the present experiments forms evidence for the existence of coordination strategies that provide an additional mechanism for temporal coordination. Our findings cannot easily be explained by motor simulation or entrainment. If coactors had simulated the other's actions based on their own performance (Ramnani and Miall 2004), we should have found equally variable and short RTs in the individual and the joint conditions because coactors would have taken their own performance as a model rather than modulating their own performance.

The pattern observed when participants intended to coordinate their actions is also not readily explained by entrainment. First, the tendency to entrain is attributed to a coupling of oscillators that requires continuous information exchange between individuals. For instance, two people swing hand-held pendulums or rock in rocking chairs with the same frequency only when they look at each other and not when the coactor acts in peripheral vision (Richardson et al. 2005, 2007). In the present task, participants could 
only see each other's actions in peripheral vision and they performed discrete non-rhythmic rather than continuous rhythmic actions.

Second, the findings of the sequential coordination task (Experiment 3) cannot be accounted for by people's tendency to synchronize, because the task required acting not at the same time but in close temporal succession. We found the same link between response variability and asynchrony in the synchronous and in the sequential coordination task, suggesting that a common mechanism underlies both forms of temporal coordination. Third, whereas entrainment occurs both in intentional and unintentional coordination contexts (Richardson et al. 2007), we found a different pattern depending on whether or not people intended to coordinate. Whereas in the intentional coordination condition, reduced variability was associated with better coordination performance, in the unintentional coordination condition only response speed correlated with asynchrony. Moreover, people were more synchronized in the intentional condition compared with the unintentional condition, indicating that there was a top-down modulation on motor performance.

The findings from the unintentional condition in Experiment 2 demonstrate that synchronization can also occur when coactors do not intend to coordinate their actions. It seems that participants' tendency to emphasize speed contributed to this emergent coordination. One could speculate that participants more readily fell into a common rhythm when they both responded quickly, which increased the probability of their responses occurring at the same time. Whether this finding can be explained in terms of coupled oscillator models within a dynamical system framework remains to be seen.

Although we argue that in the intentional coordination tasks predictability was used as a coordination strategy, this does not imply that people relied on explicit knowledge of the relation between variability and coordination performance or that they were consciously aware of their strategic use of this relation. In fact, it is likely that people did not plan to modify their own behavior in this particular way to make it easier for their coactor to predict their upcoming actions. Rather, they may have formed a general intention to be as coordinated as possible, triggering a particular modus operandi of the action system that rendered the timing of actions less variable. The use of strategies that are not necessarily in explicit awareness is well supported by game theory research, where certain strategies such as "tit for tat" can be observed even when individuals are not explicitly aware of using these strategies (Axelrod and Hamilton 1981; Brosnan et al. 2010). Further research is needed to test whether the coordination strategy we have identified in the present study is also used in more complex tasks and how exactly the intention to coordinate interacts with processes in the action system.
Our findings contribute to the understanding of joint action by demonstrating that the strategy to make oneself predictable is not only used in Schelling game-like situations where individuals need to coordinate their choices without being able to communicate (Clark 1996; Schelling 1960) but also in joint tasks requiring close temporal coordination. In the absence of continuous feedback about a coactor's actions, reducing the variability of one's own actions is a simple yet powerful mechanism for achieving coordination as it helps coactors to establish procedural common ground (Clark 1996) that is required for successful coordination.

Open Access This article is distributed under the terms of the Creative Commons Attribution Noncommercial License which permits any noncommercial use, distribution, and reproduction in any medium, provided the original author(s) and source are credited.

\section{References}

Aiello JR, Douthitt EA (2001) Social facilitation from Triplett to electronic performance monitoring. Group Dynam Theory Res Pract 5:163-180

Atmaca S, Sebanz N, Prinz W, Knoblich G (2008) Action co-representation: the joint SNARC effect. Soc Neurosci 3:410-420

Atmaca S, Sebanz N, Knoblich G (2011) The joint Flanker effect: sharing tasks with real and imagined co-actors. Exp Brain Res. doi:10.1007/s00221-011-2709-9

Axelrod R, Hamilton WD (1981) The evolution of cooperation. Science 211:1390-1396

Brennan SE, Clark HH (1996) Conceptual pacts and lexical choice in conversation. J Exp Psychol Learn Mem Cognit 22:1482-1493

Brennan SE, Hanna JE (2009) Partner-specific adaptation in dialog. Top Cognit Sci 1:274-291

Brosnan SF, Salwiczek L, Bshary R (2010) The interplay of cognition and cooperation. Phil Trans R Soc B 365:2699-2710

Clark H (1996) Using language. Cambridge University Press, Cambridge

Garrod S, Pickering MJ (2004) Why is conversation so easy? Trends Cognit Sci 8:8-11

Garrod S, Pickering MJ (2009) Joint action, interactive alignment, and dialog. Top Cognit Sci 1:292-304

Keller PE, Knoblich G, Repp BH (2007) Pianists duet better when they play with themselves: on the possible role of action simulation in synchronization. Conscious Cognition 16:102-111

Knoblich G, Jordan JS (2003) Action coordination in groups and individuals: learning anticipatory control. J Exp Psychol Learn Mem Cognit 29:1006-1016

Konvalinka I, Vuust P, Roepstorff A, Frith CD (2010) Follow you, follow me: continuous mutual prediction and adaptation in joint tapping. Quart J Exp Psychol 63:2220-2230

Kornblum S, Hasbroucq T, Osman A (1990) Dimensional overlap: cognitive basis for stimulus-response congruency - a model and taxonomy. Psychol Rev 97:253-270

Loehr JD, Palmer C (submitted) Temporal coordination between performing musicians

Loftus G, Masson MEJ (1994) Using confidence intervals in withinsubject designs. Psychon Bull Rev 1:476-490

Milanese N, Iani C, Rubichi S (2010) Shared learning shapes human performance: transfer effects in task sharing. Cognition 116:15-22

Ramnani N, Miall RC (2004) A system in the human brain for predicting the actions of others. Nat Neurosci 7:85-90 
Repp BH (2005) Sensorimotor synchronization: a review of the tapping literature. Psychon Bull Rev 12:969-992

Richardson MJ, Marsh KL, Schmidt RC (2005) Effects of visual and verbal information on unintentional interpersonal coordination. J Exp Psychol Hum Percept Perform 31:62-79

Richardson MJ, Marsh KL, Isenhower R, Goodman J, Schmidt RC (2007) Rocking together: dynamics of intentional and unintentional interpersonal coordination. Hum Mov Sci 26:867-891

Schelling TC (1960) The strategy of conflict. Harvard University Press, Cambridge

Schmidt RC, Bienvenu M, Fitzpatrick PA, Amazeen PG (1998) A comparison of intra- and interpersonal interlimb coordination: coordination breakdowns and coupling strength. J Exp Psychol Hum Percept Perform 24:884-900

Sebanz N, Knoblich G (2009) Prediction in joint action: what, when, and where. Top Cognit Sci 1:353-367

Sebanz N, Bekkering H, Knoblich G (2006) Joint action: bodies and minds moving together. Trends Cognit Sci 10:70-76

Simon JR (1990) The effects of an irrelevant directional cue on human information processing. In: Proctor RW, Reeve TG (eds) Stimu- lus-response congruency: an integrated perspective. Amsterdam, North-Holland, pp 31-86

van der Wel RPRD, Knoblich G, Sebanz N (2011) Let the force be with us: Dyads exploit haptic coupling for coordination. J Exp Psychol Hum Percept Perform

Van Ulzen NR, Lamoth CJC, Daffertshofer A, Semin GR, Beek PJ (2008) Characteristics of instructed and uninstructed interpersonal coordination while walking side-by-side. Neurosci Lett 432:88-93

Vesper C, Butterfill S, Knoblich G, Sebanz N (2010) A minimal architecture for joint action. Neural Netw 23:998-1003

Wagenmakers EJ, Brown S (2007) On the linear relation between the mean and the standard deviation of a response time distribution. Psychol Rev 114:830-841

Wolpert DM, Doya K, Kawato M (2003) A unifying computational framework for motor control and interaction. Phil Transact Roy Soc Lond B 358:593-602

Zajonc RB (1965) Social facilitation. Sci 149:269-274 